\title{
Massa muscular em militares da reserva: comparação de variáveis musculares e composição corporal
}

\section{Muscle mass in reserve soldiers: comparison of muscle variables and body composition}

\author{
Michael Hanemann Fagundes, Paulo Roberto Nessi Carnacini Custódio, \\ Patrik Nepomuceno, Maiara Helena Rusch, Hildegard Hedwig Pohl
}

Como citar este artigo: FACUNDES, MICHAEL H.; CUSTÓDIO, PAULO R. N. C.; NEPOMUCENO, PATRIK; RUSCH, MAIARA H.; POHL, HILDECARD H.; Massa muscular em militares da reserva: comparação de variáveis musculares e composição corporal. Revista Saúde (Sta. Maria). 2020; 46 (2).

Autor correspondente:

Nome: Michael Hanemann Fagundes

E-mail: maike317@hotmail.com

Telefone: (51) 3717-7604

Formação Profissional: Formado

em Educação Física pela

Universidade de Santa Cruz do

Sul (UNISC), Santa Cruz do Sul,

RS, Brasil

Filiação Institucional: Universidade de Santa Cruz do Sul

Endereço para correspondência:

Av. Independência $n^{\circ}: 2293$

Bairro: Universitário

Cidade: Santa Cruz do Sul

Estado: Rio Grande do Sul

CEP: 96.815-900

Data de Submissão:

30/03/2020

Data de aceite:

26/07/2020

Conflito de Interesse: Não há conflito de interesse

\section{(cc) $\mathrm{BY}-\mathrm{NC}-\mathrm{ND}$}

\section{RESUMO}

0 processo de envelhecimento leva à dificuldade do corpo manter seu equilibrio homeostático e isso, sob circunstâncias de sobrecarga funcional, gera déficit de alguns sistemas corporais, podendo evoluir com comprometimento de determinadas funções, especialmente pela perda de massa muscular. Objetivo: identificar se há relação entre a prática de atividade física e a massa muscular esquelética e composição corporal de militares da reserva. Métodos: trata-se de um estudo descritivo, comparativo e retrospectivo, em que foram avaliados 33 militares da reserva, divididos em dois grupos praticantes de atividade física e não praticantes. Os instrumentos utilizados foram: questionário de estilo de vida, estadiômetro acoplado à balança, fita antropométrica e 0 equipamento de bioimpedância. A partir destes instrumentos obteve-se variáveis demográficas, circunferência da cintura, massa muscular esquelética, índice de massa corporal, percentual de gordura e o índice de músculo esquelético. Resultados: Aidade média dos participantes do estudo foi de 58,4 anos, sendo que a maioria eram praticantes de atividade física e casados. Em relação a atividade física não foram observadas diferenças quanto a dados sociodemográficos, de composição corporal e massa muscular. Conclusão: A maior parte dos militares da reserva eram fisicamente ativos. Em relação a prática de atividade física é possível concluir que não há diferença entre praticantes e não praticantes no que se refere a variáveis sociodemográficas e estilo de vida. Além disso, não foram observadas diferenças estatisticamente significativas entre os grupos quanto às variáveis musculares e composição corporal.

PALAVRAS-CHAVE: Envelhecimento; Músculo esquelético; Militares.

\section{ABSTRACT}

The aging process leads to the body's difficulty in maintaining its homeostatic balance and this, under circumstances of functional overload, generates deficits in some body systems, which may evolve with impairment of certain functions, especially due to the loss of muscle mass. Objective: to identify if there is a relationship between physical activity and skeletal muscle mass and body composition of reserve military personnel. Methods: this is a descriptive, comparative and retrospective study, in which 33 reserve military personnel were evaluated, divided into two groups who practice physical activity and non-practitioners. The instruments used were: lifestyle questionnaire, stadiometer attached to the scale, anthropometric tape and bioimpedance equipment. From these instruments, demographic variables, waist circumference, skeletal muscle mass, body mass index, fat percentage and skeletal muscle index were obtained. Results: The average age of the study participants was 58.4 years, most of whom were engaged in physical activity and were married. About physical activity, there were no differences regarding sociodemographic data, body composition and muscle mass. Conclusion: Most of the reserve military personnel were physically active. Regarding the practice of physical activity, it is possible to conclude that there is no difference between practitioners and non-practitioners with regard to sociodemographic variables and lifestyle. In addition, there were no statistically significant differences between groups regarding muscle variables and body composition. 


\section{INTRODUÇÃO}

Com o processo de envelhecimento, o corpo acaba não sendo capaz de manter seu equilíbrio homeostático e, isso sob circunstâncias de sobrecarga funcional, gera déficit de alguns sistemas corporais podendo evoluir para situações de incapacidade, com comprometimento de determinadas funções. No envelhecimento ocorrem alterações tanto fisiológicas quanto psíquicas e/ou sociais, que de certa forma, podem ou não interferir na qualidade de vida do indivíduo.

As perdas físicas e funcionais decorrentes do envelhecimento podem ser acentuadas pela inatividade física². Entre estas perdas estão as várias alterações que ocorrem no músculo esquelético. A redução da massa muscular associada à idade é um dos principais contribuintes no comprometimento osteomuscular ${ }^{3}$. Neste sentido, Oliveira ${ }^{4}$ diz que a prática regular de atividade física minimiza os efeitos fisiológicos do envelhecimento e também melhora aspectos psicológicos e cognitivos, reduzindo o desenvolvimento e avanço de doenças crônicas e condições de incapacidades. De forma mais específica Papa, Dong e Hassan ${ }^{3}$ recomendam que os déficits da função muscular associados à idade podem ser melhorados pelo treinamento de resistência. Assim, o principal objetivo na minimização da perda de massa muscular é retardar a progressão. Para tanto o exercício físico, para a população em geral e idosa, em particular, pode beneficiar diferentes aspectos da saúde do praticante e promover uma melhora na aptidão física.

No âmbito das forças armadas, treinamento físico é relevante e extremamente importante para a saúde e condicionamento físico dos militares. Uma vez que estes profissionais precisam apresentar boa aptidão física para a realização das demandas diárias o que torna importante a prática de atividades físicas orientadas, acompanhamento médico e de índices e medidas que possam estabelecer o nível de condicionamento físico 5 .

As Forças Armadas de maneira global reconhecem a importância do treinamento de exercícios militares na preparação, liderança e atividade de suas tropas. Nas diferentes batalhas em que o exército americano se fez presente, foi ratificado que a aptidão física desempenha um importante papel no campo de batalha. $\mathrm{O}$ exército canadense afirma que a atividade física aumenta o estado de alerta, a autoestima e o vigor nas operações. Portanto, uma boa aptidão física proporciona enfrentar melhor situações estressantes intrínsecas à atividade militar ${ }^{6}$.

Do mesmo modo, Vargas, Moleta e Pilatti ${ }^{7}$, citam que, entre as prioridades no programa de instrução militar anual do Exército Brasileiro está o treinamento físico militar. Esta atividade é prevista para todos, ao longo da carreira, com disponibilidade de uma hora e meia durante as oito horas diárias de trabalho. Todos os exercícios físicos praticados pelos militares estão regulamentados no manual de instrução, assim, é possível que militares da reserva se beneficiem dos ganhos adquiridos por meio do exercício físico ao longo da carreira. Ao considerar a rotina de exercícios físicos que integra o cotidiano do militar, o objetivo deste estudo foi identificar se há relação entre a prática de atividade física e a massa muscular esquelética e composição corporal de militares da reserva. 


\section{MÉTODO}

Trata-se de pesquisa descritiva, comparativa e retrospectiva, realizada a partir do banco de dados do projeto "Prevalência de sarcopenia de um grupo de militares de um batalhão do exército brasileiro", aprovado pelo Comitê de Ética em Pesquisa da instituição com o CAAE 01516218.2.0000.5343. A amostra do estudo foi composta de 33 militares da reserva remunerada, cadastrados na Seção de Inativos e Pensionistas, localizado no município de Santa Cruz do Sul, RS.

Para a análise das variáveis, os militares foram divididos em dois grupos, o primeiro grupo praticante de atividade física e o outro não praticante de atividade física. Os dados sociodemográficos e de estilo de vida (idade, estado civil, atividade física, horas de sono e graduação militar) foram obtidos a partir de questionário; as variáveis foram classificadas da seguinte forma: idade (em anos); estado civil (casado/outros); prática de atividade física (sim/não); horas de sono diária (menos 7h/mais de 7h); graduação militar (coronel/major/capitão/tenente/sargento) e classe socioeconômica (A/B/C/D/E).

A estatura e peso foram obtidos por meio de balança analógica e estadiômetro, para cálculo do índice de massa corporal (IMC) foi utilizada a fórmula peso $(\mathrm{kg}) /$ altura $\left(\mathrm{m}^{2}\right)$ e a circunferência da cintura $(\mathrm{CC})$ foi obtida por meio de fita antropométrica no ponto médio entre a última costela e a crista ilíaca. A avaliação de composição corporal foi realizada através de bioimpedanciometria, por meio do aparelho InBody 720 (composição corporal analyze, Biospace CO. Ltda); foram obtidas as variáveis: massa muscular esquelética $(\mathrm{MME})$ e percentual de gordura $(\% \mathrm{G})$. A partir destas variáveis foi calculado o índice de musculo esquelético (IME), que consiste na razão entre o percentual da MME com o percentual da massa corporal (MC) do indivíduo (MME/MCx100).

Os dados foram analisados por estatística descritiva e analítica por meio do programa IBM SPSS for Windows (v.23.0), utilizando média e desvio padrão e frequência e percentual. A normalidade dos dados foi verificada pelo Teste de Shapiro-Wilk; a comparação de médias entre os grupos foi realizada através do Teste $t$ de Student para amostras independentes (variáveis paramétricas) e U de Mann-Whitney (variáveis não-paramétricas). Para comparação entre os grupos de variáveis categóricas foi utilizado o Teste de Qui-Quadrado ou Exato de Fischer, considerando p < 0,05.

\section{RESULTADOS}

A partir da análise dos resultados observou-se que a idade média dos participantes do estudo foi de 58,4 anos, sendo que a maioria eram praticantes de atividade física, casados, pertencentes as classes socioeconômicas C e D; na tabela 1 estão as médias gerais da composição corporal e massa muscular dos avaliados. 
Tabela 1. Caracterização dos militares quanto aos dados demográficos, composição corporal e massa muscular esquelética.

\begin{tabular}{ll}
\hline Variável & $\mathrm{n}=33$ \\
& $\mathrm{n}(\%)$ \\
\hline Idade† & $58,4(6,8)$ \\
Estado Civil & \\
Casado & $25(76)$ \\
Outros & $8(24)$ \\
Classificação Socioeconômica ${ }^{a}$ & \\
Classe B & $10(34)$ \\
Classe C e D & $19(66)$ \\
Graduação & \\
Coronel & $2(6)$ \\
Major & $1(3)$ \\
Capitão & $10(30)$ \\
Tenente & $7(21)$ \\
Sargento & $13(39)$ \\
Tempo de Sono & \\
Menos de 7h & \\
Mais de 7h & $15(47)$ \\
Prática de Atividade Física & $17(53)$ \\
Sim & \\
Não & $23(70)$ \\
Índice de Massa Corporal† & $10(30)$ \\
Percentual de Gordura† & $26,9(3,9)$ \\
Massa Muscular Esquelética† & $92,2(9,9)$ \\
Índice Músculo Esquelético† & $24,1(6,8)$ \\
\hline & $34,5(4,8)$ \\
\hline médiância da Cintura† & $42,7(3,8)$ \\
\hline
\end{tabular}

†: média (desvio padrão); n: frequência absoluta; \%: frequência relativa; a: 4 missing; b: 1 missing.

A tabela 2 apresenta a comparação entre os grupos praticante e não praticante de atividade física em relação a variáveis sociodemográficas e de estilo de vida. Não foram observadas diferenças quanto aos grupos em relação a idade, estado civil, classe socioeconômica, graduação militar e tempo de sono. 
Tabela 2. Comparação de variáveis sociodemográficas e de estilo de vida de acordo com a prática de atividade física.

\begin{tabular}{|c|c|c|c|}
\hline \multirow[t]{4}{*}{ Variável } & \multicolumn{2}{|c|}{ Prática de Atividade Física } & \multirow[t]{4}{*}{$p$} \\
\hline & $\operatorname{Sim}$ & Não & \\
\hline & $(n=23)$ & $(n=10)$ & \\
\hline & $\mathrm{n}(\%)$ & $\mathrm{n}(\%)$ & \\
\hline Idade† & $58,2 \pm 6,0$ & $58,7 \pm 8,9$ & 0,856 \\
\hline \multicolumn{4}{|l|}{ Estado Civil } \\
\hline Casado & $16(64)$ & $9(36)$ & 0,382 \\
\hline Outros & $7(87)$ & $1(13)$ & \\
\hline \multicolumn{4}{|c|}{ Classificação Socioeconômica ${ }^{a}$} \\
\hline Classe B & $8(80)$ & $2(20)$ & 0,431 \\
\hline Classe C e D & $12(63)$ & $7(37)$ & \\
\hline \multicolumn{4}{|l|}{ Graduação } \\
\hline Coronel & $1(50)$ & $1(50)$ & 0,786 \\
\hline Major & $1(100)$ & - & \\
\hline Capitão & $8(80)$ & $2(20)$ & \\
\hline Tenente & $5(71)$ & $2(29)$ & \\
\hline Sargento & $8(62)$ & $5(38)$ & \\
\hline \multicolumn{4}{|c|}{ Tempo de Sonob } \\
\hline Menos de $7 \mathrm{~h}$ & $9(60)$ & $6(40)$ & 0,450 \\
\hline Mais de $7 \mathrm{~h}$ & $13(76)$ & $4(24)$ & \\
\hline
\end{tabular}

Na tabela 3 estão expressas as variáveis musculares e de composição corporal relacionadas a prática ou não de atividade física. Os resultados demonstram que não houve diferença estatística entre os grupos praticante e não praticante. Isso sugere que prática de atividade física ao longo da carreira militar ativa pode ter assegurado resultados positivos na MME, IME, IMC, CC e \%G dos militares da reserva.

Tabela 3. Comparação das variáveis musculares e de composição corporal de acordo com a prática de atividade física.

\begin{tabular}{llll}
\hline Variável & \multicolumn{2}{l}{ Prática de Atividade Física } & p \\
& $\begin{array}{l}\text { Sim } \\
\text { (n=23) }\end{array}$ & $\begin{array}{l}\text { Não } \\
\text { (n=10) }\end{array}$ & \\
& Média (DP) & Média (DP) & \\
\hline Massa Muscular Esquelética & $35,0(4,7)$ & $33,3(5,1)$ & 0,259 \\
Índice de Musculo Esquelético & $42,8(3,5)$ & $42,4(4,8)$ & 0,882 \\
Índice de Massa Corporal & $26,9(3,2)$ & $26,7(4,4)$ & 0,681 \\
Circunferência da Cintura & $92,7(9,2)$ & $91,1(12,0)$ & 0,793 \\
Percentual de Gordura & $24,3(5,9)$ & $23,6(8,7)$ & 0,769 \\
\hline
\end{tabular}

n: frequência absoluta; DP: desvio padrão; p: nível de significância. 


\section{DISCUSSÃO}

O presente estudo objetivou identificar a relação entre a prática de atividade física e a massa muscular esquelética e composição corporal de militares da reserva do Regimento Gomes Carneiro de Santa Cruz do Sul. A partir dos resultados analisados, foi possível observar que não há relação entre a prática de atividade física e variáveis musculares e composição corporal.

A redução da massa muscular esquelética com o avanço da idade está entre as perdas físicas e funcionais decorrentes do envelhecimento, em nosso estudo, praticantes e não praticantes de atividade física apresentavam média de idade semelhantes (menos de 60 anos). Alencar et al. ${ }^{10}$ apontaram que ocorrem maiores perdas de MME nas faixas etárias acima de 60 anos e a prática de atividade física pode ser um fator protetor, entretanto tais achados não foram observados em nosso estudo, fato que pode ser devido aos avaliados apresentarem menos de 60 anos. Essas perdas não têm como único fator o declínio da idade, pois é gradual no funcionamento de todos os sistemas ao longo do envelhecimento, com ápice a partir dos 70 anos, mas também o sedentarismo, a baixa capacidade física e outras comorbidades, fatores que podem ser atenuados devido a atividade militar.

Pícoli, Figueiredo e Patrizzi ${ }^{10}$, em estudo realizado com 48 indivíduos de ambos sexos, subdivididos em 4 grupos por faixa etária (G1 - 11 a 18 anos, G2- 20 a 26 anos, G3 - 45 a 60 anos e G4 - 66-82 anos), constataram que a força muscular tende a aumentar com a idade (G1, G2 e G3), mas, ao comparar os grupos G3 e G4 foi observada redução da força muscular no grupo do intervalo etário dos 66 aos 82 anos, fator este, que pode estar relacionado com a diminuição da massa muscular ${ }^{11}$, além disso, a prática de atividade física pode aumentar/manter a massa muscular, a medida que praticantes apresentam maior massa muscular, entre os militares avaliados, não foram observadas diferenças na massa muscular e composição corporal, indicando que a atividade física após a saída do exército não confere benefício adicional a esses.

Os resultados da MME e IME relacionados a prática ou não de atividade física não apresentaram significância estatística. Contudo, verificou-se que os valores da MME e o IME foram maiores no grupo praticante de atividade física. Estudo de Guimarães et al. ${ }^{12}$, que comparou o risco de queda em 20 idosos praticantes e 20 não praticantes de atividade física, observaram que $95 \%$ dos praticantes apresentavam baixo risco de queda, enquanto $85 \%$ dos não praticantes foram classificados com risco médio e alto de quedas.

Já o estudo de Silva et al. ${ }^{3}$, apontou que independente da modalidade de atividade física praticada, o idoso terá benefícios importantes para um envelhecimento independente e saudável. Todavia, quando a atividade é realizada como exercício físico de forma sistematizado, pode-se alcançar benefícios melhores, tais resultados não são identificados em militares, já que não foram observadas diferenças quanto aos grupos praticante e não praticante de atividade física.

Em relação à atividade física um estudo realizado por Ferreira et al. ${ }^{13}$ analisou 50 idosos praticantes de atividade física, subdivididos em dois grupos (G1 e G2), G1 com 32 indivíduos praticantes em nível moderado e G2 com 18 
participantes em nível baixo. O resultado deste estudo, apontou que o $\mathrm{G} 1$, apresentou valores de $\% \mathrm{G}$ significativamente menores do que o G2, indicando que a intensidade da atividade física praticada teve influência no \%G. Em nosso estudo, não foi possível observar diferenças na composição corporal e estado nutricional de militares praticantes e não praticantes, reforçando que a atividade militar ao longo da vida beneficia aqueles que não praticam atividade física após a aposentadoria.

Almeida e Silva ${ }^{14}$, avaliaram a função muscular e a composição corporal de 15 idosos fisicamente ativos em um treinamento combinado de oito semanas (força, equilíbrio, flexibilidade e resistência aeróbica) e observaram uma redução $1,27 \%$ do \%G entre o pré e pós-treinamento; indicando que a atividade física pode contribuir na melhora da composição corporal de idosos, no presente estudo não se observou relação entre \%G e IMC e CC e atividade física.

No estudo de Barbosa e Costa $^{15}$, foram avaliadas 20 idosas, divididas em dois grupos. G1 com 10 idosas praticantes de voleibol e G2 com 10 idosas sedentárias. Neste estudo os autores constataram que o G1 tinha \%G menor em relação ao G2. Do mesmo modo encontraram valores de massa magra superior no G1 em relação ao G2, indicando uma relação entre as variáveis, \%G e massa magra, tais resultados não foram identificados em nosso estudo, podendo estar relacionado a idade dos militares e também aos benefícios adquiridos pela prática de atividade física ao longo da carreira militar.

Em síntese, no que tange a pratica de atividade física, não foram encontrados resultados estatísticos significativos nas variáveis analisadas, esses resultados podem estar relacionados ao fato de que os participantes realizaram atividade física intensa durante toda sua vida como militar e logram benefícios do exercício físico mesmo após a aposentadoria; além disso, grande parte dos militares mantiveram a prática mesmo após a saída do exército.

Este estudo apresenta algumas limitações, bem como, alguns pontos positivos. Como aspectos negativos, destaca-se que o estudo apresenta limitações inerentes ao corte transversal. Enquanto no âmbito positivo destacamos o ineditismo do estudo deste tema com militares, um grupo que tem a aptidão física como condição inerente a carreira militar, o que permite estudar as variáveis musculares e antropométricas e sua relação com prática de atividade física.

\section{CONSIDERAÇÕES FINAIS}

Os militares da reserva apresentaram média de idade de 58,4 anos e a maior parte era fisicamente ativa. Em relação a prática de atividade física é possível concluir que não há diferença entre praticantes e não praticantes no que se refere a idade, estado civil, classe socioeconômica, graduação militar e tempo de sono. Além disso, não foram observadas diferenças estatisticamente significativas entre os grupos quanto às variáveis musculares e composição corporal, diante disso, acredita-se que a prática de atividade física durante a carreira militar pode estar relacionada a esses resultados. 


\section{REFERÊNCIAS}

1. Bernardi DF, Reis MAS, Lopes NB. O tratamento da sarcopenia através do exercício de força na prevenção de quedas em idosos: revisão de literatura. Ensaios e Ciência: Ciências Biológicas, Agrárias e da Saúde. 2008;xxi(2):197-213.

2. Hansen D, Rückert TK, Rosa CB, Garces SBB, Figueiró MF, Myskiw JC, et al. Nível de atividade física e risco de quedas em idosos da comunidade. Saúde (Santa Maria). 2016;42(2):157-166.

3. Papa EV, Dong X, Hassan M. Skeletal Muscle Function Deficits in the Elderly: Current Perspectives on Resistance Training. J Nat Sci. 2017;3(1):e272.

4. Oliveira DV, Silva TPS; Scherer FC, Nascimento Júnior JRA, Antunes MD. O tipo de exercício físico interfere na frequência da prática de atividade física, comportamento sedentário, composição corporal e estado nutricional do idoso. Rev Bras Nutr Esport. 2019;13(77):3-16.

5. Silva MF, Goulart NBA, Lanferdini FJ, Marcon M, Dias CP. Relação entre os níveis de atividade física e qualidade de vida de idosos sedentários e fisicamente ativos. Rev Bras Geriatr Geronto. 2012;15(4):635-642.

6. CASTRO, Celso. In Corpore Sano - os militares e a introdução da educação física no Brasil. Antropolítica. 1997;2:61-78.

7. Vargas LM, Moleta T, Pilatti, LA. Diferença da aptidão física relacionada ao desempenho entre soldados de elite e convencionais do exército brasileiro. Conexões. 2013;11(2):148-167.

8. American College Of Sports Medicine. Manual do ACMS para avaliação da aptidão física relacionada à saúde. Rio de Janeiro: Guanabara Koogan; 2006.

9. Alencar MSS, Melo MTSM, Sousa RC, Campos CMF, Meneses AV, Sepúlveda L. et al. Perdas de massa muscular e adiposa após institucionalização: atenção aos mais idosos. Geriatr Gerontol Aging. 2015;9(4):150-155. 
10. Pícoli TS, Figueiredo LL, Patrizzi LJ. Sarcopenia e envelhecimento. Fisioter Mov. 2011;24(3):455-62.

11. Souza CF, Vieira MCA, Nascimento RA, Moreira MA, Câmara SMA, Maciel ACC. Relação entre força e massa muscular em mulheres de meia-idade e idosas: um estudo transversal. Rev. Bras. Geriatr. Gerontol., Rio de Janeiro, 2017;20(5):661-670.

12. Guimarães LHCT, Galdino DCA, Martins FLM, Vitorino DFM, Pereira KL, Carvalho, EM. Comparação da propensão de quedas entre idosos que praticam atividade física e idosos sedentários. Rev Neurociênc.2004;12(2):68-72.

13. Ferreira APS, Picolli T, Bordin A, Rech A, Poeta J, Tiggemann CL, et al. Baixos níveis de atividade física estão associados a prejuízos no perfil lipídico e aumento do percentual de gordura de indivíduos idosos. Rev Bras Ci e Mov. 2015;23(3):135-142.

14. Almeida DKS; Silva FOC. A função muscular e a composição corporal na qualidade de vida do idoso: Efeitos de um programa de 8 semanas de treinamento combinado. Rev Bras Presc e Fisiol do Exerc. 2016;10(60):504510.

15. Barbosa CR., Costa DDA. Análise comparativa da composição corporal de idosas praticantes da modalidade voleibol e sedentárias [trabalho de conclusão de curso]. Uberaba: Universidade de Uberaba. Curso de Educação Física, 2018. 